\title{
Transfusion-related acute lung injury; clinical perspectives
}

\author{
Jeongmin $\mathrm{Kim}^{1,2}$ and Sungwon $\mathrm{Na}^{1,2}$ \\ ${ }^{1}$ Department of Anesthesiology and Pain Medicine, ${ }^{2}$ Anesthesia and Pain Research Institute, Yonsei University \\ College of Medicine, Seoul, Korea
}

Transfusion-related acute lung injury (TRALI) was introduced in 1983 to describe a clinical syndrome seen within $6 \mathrm{~h}$ of a plasma-containing blood products transfusion. TRALI is a rare transfusion complication; however, the FDA has suggested that TRALI is the leading cause of transfusion-related mortality. Understanding the pathogenesis of TRALI will facilitate adopting preventive strategies, such as deferring high plasma volume female product donors. This review outlines the clinical features, pathogenesis, treatment, and prevention of TRALI.

Key Words: Acute lung injury, Diagnosis, Etiology, Prevention and control, Prognosis, Transfusion reaction.

Since transfusion was introduced, the associated risks, including transfusion-transmitted infection and incompatibility, have decreased with improved quality [1]. Although the frequency of many complications has decreased, transfusionrelated acute lung injury (TRALI) has become one of the major complications associated with transfusion. TRALI is a potentially fatal blood-product transfusion complication that presents as acute hypoxemia and noncardiogenic pulmonary edema.

The first case of TRALI was reported in the 1950s [2]. Although TRALI has been termed a pulmonary hypersensitivity reaction, pulmonary infiltrate associated with transfusion reaction, and fulminating noncardiogenic pulmonary edema [3], the diagnostic term TRALI was first suggested by Popovsky et al. [4]

Corresponding author: Sungwon Na, M.D., Ph.D.

Department of Anesthesiology and Pain Medicine, Anesthesia and

Pain Research Institute, Yonsei University College of Medicine, 50

Yonsei-ro, Seodaemun-gu, Seoul 120-752, Korea

Tel: 82-2-2228-2420, Fax: 82-2-312-7185

E-mail: nswksj@yuhs.ac

Received: December 30, 2014.

Revised: February 6, 2015.

Accepted: February 9, 2015.

Korean J Anesthesiol 2015 April 68(2): 101-105

http://dx.doi.org/10.4097/kjae.2015.68.2.101 in 1983.

\section{Definition and Epidemiology}

TRALI is the leading cause of transfusion-related mortality in the United States. TRALI was defined based on clinical and radiological parameters at the 2004 Consensus Conference, as newly developed acute lung injury (ALI)/acute respiratory distress syndrome (ARDS) within $6 \mathrm{~h}$ of a blood product transfusion (Table 1) [3]. The $6 \mathrm{~h}$ time window was chosen based on the opinion of an expert panel. Suspected TRALI was defined as fulfillment of the definition of TRALI without another ALI risk factor. Because the characteristics of TRALI are difficult to distinguish from those of ALI due to other causes, possible TRALI has been suggested to allow for the presence of other ALI risk factors. The conventional definition of TRALI included cases of ALI that develop within $6 \mathrm{~h}$ of transfusion, but transfusion is an ALI risk factor for 6-72 h. Thus, delayed TRALI includes delayed onset TRALI after transfusion [5].

TRALI occurs in up to $15 \%$ of transfused patients [6], but other reports estimate the incidence of TRALI at approximately $0.1 \%$ of transfused patients $[7,8]$.

Although the true incidence of TRALI is unknown, it is likely under-diagnosed and under-estimated, primarily due to poor recognition of the disease [8]. Several studies have reported inci-

(c) This is an open-access article distributed under the terms of the Creative Commons Attribution Non-Commercial License (http://creativecommons.org/ licenses/by-nc/3.0/), which permits unrestricted non-commercial use, distribution, and reproduction in any medium, provided the original work is properly cited. 
Table 1. Definition of TRALI

Suspected TRALI

Acute onset within $6 \mathrm{~h}$ of blood transfusion

$\mathrm{PaO}_{2} / \mathrm{FIO}_{2}<300 \mathrm{mmHg}$, or worsening of $\mathrm{P} / \mathrm{F}$ ratio

Bilateral pulmonary infiltrates on chest radiograph

No sign of hydrostatic pulmonary edema (pulmonary arterial occlusion

pressure $\leq 18 \mathrm{mmHg}$ or central venous pressure $\leq 15 \mathrm{mmHg}$ )

No other risk factor for acute lung injury

Possible TRALI

Same as for suspected TRALI, but another risk factor present for acute lung injury

Delayed TRALI

Same as for (possible) TRALI and onset within $6-72 \mathrm{~h}$ of blood transfusion

PAOP: pulmonary arterial occlusion pressure, CVP: central venous pressure, TRALI: transfusion-related acute lung injury.

dence ranges of 1 in 7,900 units of fresh frozen plasma (FFP) to 1 in 432 units of whole blood $[9,10]$. In addition, the incidence of TRALI depends on the patient population, and a higher incidence of TRALI is observed in critically ill patients [11]. Historical estimates for TRALI-associated mortality range from 5 to $8 \%$ [12], but up to $60 \%$ of critically ill patients die from TRALI [13].

\section{Risk factors}

Specific risk factors for TRALI can be divided conceptually into recipient-related risk factors and transfusion-related risk factors [14].

\section{Recipient-related risk factors}

Many comorbidities have been suggested as TRALI risk factors, including end-stage liver disease, coronary artery bypass graft, hematological malignancies, massive transfusion, mechanical ventilation, sepsis, and heavy alcohol consumption [14]. Critical illness is one of the highest risks for TRALI [11,13]. A large database review of 11 million elderly patients in the United States revealed that TRALI rates are higher in patients receiving a transfusion, including platelets or plasma [15]. TRALI incidence increase as the number of units transfused increases. A review also identified that elderly female Caucasians 65-79-yearsold with a 6-month history of post-inflammatory pulmonary fibrosis and tobacco use have a greater chance of developing TRALI [15].

\section{Transfusion-related risk factors}

Immunoglobulin [16] and stem cell preparations [17] are also associated with TRALI. A case-control study enrolled 89 patients with TRALI and 164 controls. The study revealed that a higher TRALI odds ratio (OR) is associated with plasma from female donors (OR, 4.5; 95\% confidence interval [CI], 1.85-11.2) and a greater number of transfusions (OR, 4.5; 95\% CI, 2.4-8.4). [18] Blood products including high-plasma-volume (platelet concentrates, whole blood, and FFP) have been associated with a higher incidence of TRALI [13]. High loading of anti-human neutrophil antigen antibody has also been associated with TRALI [18]. In contrast, the presence of anti-human leukocyte antigen (HLA) class II or anti-HLA class I antibodies is not associated with TRALI.

The age of a blood product has been suggested to increase the risk for TRALI [19]. Storing red blood cells (RBCs) results in a number of morphological and biochemical alterations known as RBC storage lesions. However, two studies failed to reveal blood age as a risk factor for TRALI $[13,18]$.

\section{Pathogenesis}

\section{Two-hit model}

A two-hit hypothesis has been suggested for developing TRALI [20]. The first hit involves neutrophil sequestration and priming in the pulmonary endothelium, which precedes transfusion. Endothelial cells are thought to be responsible for both neutrophil sequestration and priming. The second hit is caused by activation of the endothelium and neutrophils by mediators in the transfused blood, resulting in capillary leakage and pulmonary edema. The second hit differs in antibody-mediated TRALI and non-antibody-mediated TRALI. Non-antibodymediated TRALI is caused by the accumulation of proinflammatory mediators during storage of blood products and possibly by ageing of RBCs and platelets [21], whereas antibody-mediated TRALI or immune TRALI is caused by passive transfusion of HLA or human neutrophil antigen (HNA) and corresponding antibodies from the donor directed against recipient antigens [22]. Donor anti-leukocyte antibodies interact with recipient neutrophils, monocytes, and/or the pulmonary endothelium [14]. Bioactive lipids and other factors in transfused blood act as biological response modifiers in patients with non-antibodymediated TRALI. The proportions of antibody-mediated vs. non-antibody-mediated TRALI cases remain unclear. A systemic review showed that $86 \%$ of TRALI cases are associated with leukocyte antibodies [23].

\section{Threshold model}

The two-hit hypothesis explains the occurrence of TRALI in high-risk patients. However, this hypothesis does not fit TRALI that develops in healthy recipients; the threshold model has been proposed to explain these cases [24]. This model generally agrees that two hits are needed to develop TRALI. However, the 
threshold model assumes that TRALI can occur when the second hit is so strong that an initial priming event is not needed. In other words, a large quantity of antibody (second hit) can cause TRALI in a recipient with no previous predisposition, such as in healthy individuals.

\section{Clinical presentation and diagnosis}

TRALI presents as sudden onset of respiratory difficulties during or shortly after transfusion. Hypoxemia and lung infiltrates are detected on chest X-rays in almost all patients with TRALI, and half of patients show a pinkish, frothy sputum [25]. Tachypnea, tachycardia, and elevated airway pressure are frequently observed. Fever, hypotension, and cyanosis occur in less than one-third of patients with TRALI.

Confirming hypoxemia, obtaining a chest X-ray, and evaluating vital signs are required to diagnose TRALI.

No laboratory test is specific for diagnosing TRALI. The greatest change is a transient drop in peripheral neutrophil count (possibly due to neutrophil sequestration in the pulmonary vasculature). Leucopenia occurs in 35\% of patients after transfusion with an antibody-containing blood product [26].

TRALI should be distinguished from pulmonary edema due to other causes. Hemolytic and septic transfusion reactions present similarly to TRALI. Anaphylaxis can cause respiratory insufficiency similar to that of TRALI, but airway signs and symptoms are more common in patients with anaphylaxis. ALI/ ARDS should be distinguished from TRALI in patients with risk factors for ALI/ARDS prior to a transfusion.

Transfusion-associated circulatory overload (TACO) is another cause of transfusion-related respiratory insufficiency (Table 2) [27]. Impaired myocardial function and rapid and aggressive fluid therapy are suggested risk factors for TACO. TRALI is more likely to be associated with signs and symptoms of inflammation, including fever, hypotension, and an exudative pulmonary infiltrate. In contrast, TACO is more likely to be associated

Table 2. Distinguishing TRALI from TACO

\begin{tabular}{lll}
\hline & \multicolumn{1}{c}{ TRALI } & \multicolumn{1}{c}{ TACO } \\
\hline $\begin{array}{l}\text { Blood pressure } \\
\text { Body temperature } \\
\text { CXR }\end{array}$ & Low-normal & Normal-elevated \\
& No vascular congestion & $\begin{array}{l}\text { Normal } \\
\text { Vascular congestion, } \\
\text { pleural effusion }\end{array}$ \\
BNP & Low $(<250 \mathrm{pg} / \mathrm{ml})$ & High \\
PAOP & Low-normal & High \\
Ejection fraction & Normal function & Abnormal function \\
Response to diuretics & Inconsistent & Improved \\
Edema fluid & Transudate & Exudate \\
\hline
\end{tabular}

TRALI: transfusion-related acute lung injury, TACO: transfusionassociated cardiac overload, CXR: chest X-ray, BNP: B type natriuretic peptide, PAOP: pulmonary artery occluding pressure. with findings suggestive of cardiac dysfunction and/or volume overload.

\section{Treatment}

No specific treatment exists for this syndrome. Corticosteroids have been used for ALI/ARDS, but the results are inconsistent. Routine use of corticosteroids in patients with TRALI is not advocated, although a successful case has been reported [28].

The transfusion should be stopped immediately if TRALI is suspected. The blood bank should be notified of the event to initiate a transfusion reaction evaluation to protect further unexpected victims.

Management of TRALI is supportive. Providing oxygen supplementation is the central management approach for hypoxemia. Limited evidence is available for managing patients with TRALI using mechanical ventilation [29], and it is logical to apply a lower tidal volume in patients with ARDS from other causes. There is a case report of applying extracorporeal membrane oxygenation in a patient with severe TRALI [30].

\section{Prognosis and prevention}

Mortality due to TRALI is $5-10 \%$, in contrast to ARDS [31]. However, 90-day mortality associated with TRALI can reach $47 \%$ in critically ill populations [13]. Long-term pulmonary function in TRALI survivors seems to be similar to that of patients who have never experienced TRALI, and no apparent late complications, such as fibrosis or other structural damage to the lung parenchyma occur as a result of TRALI [13]. The clinical course of patients with TRALI is suggested by quick resolution ( 2 days) of hypoxemia.

Cases suspected of TRALI should be promptly reported to the blood bank. The blood bank should investigate all associated donors for the presence of anti-HLA and anti-HNA antibodies to identify donors with these antibodies and prevent them from future donation. This is particularly true if the donor has antiHNA 3a [32].

In addition, several general blood donor management strategies are in use to reduce the incidence of TRALI [32-38]. First, adherence to current guidelines for utilizing blood components, particularly those for plasma, is mandatory to decrease exposure risk for patients. A restrictive transfusion strategy has been associated with a lower incidence of TRALI compared with that of a liberal transfusion strategy [39]. Clifford et al. [40] reported that patients transfused with greater volumes of blood components develop perioperative TRALI/possible TRALI more frequently than that reported previously. He reported that the overall incidence of TRALI was 22.46 per 100,000 stays. Optimal transfusion guidelines should provide sufficient blood products to 
maximize clinical outcomes while avoiding adverse events, such as TRALI. However, no golden transfusion guidelines exist for all patients. Clinicians should consider many factors when deciding to transfuse anemic patients, rather than basing the decision solely on a specified laboratory level. In this respect, Carson et al. [41] demonstrated that liberal blood transfusion approach with a hemoglobin concentration threshold of 90-100 g/L does not affect mortality in a high-risk group of elderly patients with underlying cardiovascular disease or risk factors, compared with that of a restrictive transfusion strategy with a hemoglobin concentration threshold of 70-80 g/L. Therefore, the final decision to transfuse should incorporate the patient's clinical condition, co-morbidities, and the individual wishes of the patient. Second, donors with little chance to be alloimmunized to leukocytes should receive high plasma volume components (e.g., FFP, plasma frozen within $24 \mathrm{~h}$ of phlebotomy [FP-24], plasma, cryoreduced plasma, apheresis platelets, or whole blood). Third, use pooled solvent detergent-treated plasma as an alternative to FFP. Fourth, test for anti-HLA antibodies in pregnant donors before apheresis of platelets or plasma. In particular, several management strategies including deferral of multiparous female donors are in use to reduce the incidence of TRALI [42].

\section{References}

1. Bolton-Maggs PH, Cohen H. Serious Hazards of Transfusion (SHOT) haemovigilance and progress is improving transfusion safety. Br J Haematol 2013; 163: 303-14.

2. Brittingham TE. Immunologic studies on leukocytes. Vox Sang 1957; 2: 242-8.

3. Toy P, Popovsky MA, Abraham E, Ambruso DR, Holness LG, Kopko PM, et al. Transfusion-related acute lung injury: definition and review. Crit Care Med 2005; 33: 721-6.

4. Popovsky MA, Abel MD, Moore SB. Transfusion-related acute lung injury associated with passive transfer of antileukocyte antibodies. Am Rev Respir Dis 1983; 128: 185-9.

5. Vlaar AP, Juffermans NP. Transfusion-related acute lung injury: a clinical review. Lancet 2013; 382: 984-94.

6. Benson AB, Austin GL, Berg M, McFann KK, Thomas S, Ramirez G, et al. Transfusion-related acute lung injury in ICU patients admitted with gastrointestinal bleeding. Intensive Care Med 2010; 36: 1710-7.

7. Rana R, Fernández-Pérez ER, Khan SA, Rana S, Winters JL, Lesnick TG, et al. Transfusion-related acute lung injury and pulmonary edema in critically ill patients: a retrospective study. Transfusion 2006; 46: 1478-83.

8. Silliman CC, Boshkov LK, Mehdizadehkashi Z, Elzi DJ, Dickey WO, Podlosky L, et al. Transfusion-related acute lung injury: epidemiology and a prospective analysis of etiologic factors. Blood 2003; 101: 454-62.

9. Wallis JP, Lubenko A, Wells AW, Chapman CE. Single hospital experience of TRALI. Transfusion 2003; 43: 1053-9.

10. Jaworski K, Maślanka K, Kosior DA. Transfusion-related acute lung injury: a dangerous and underdiagnosed noncardiogenic pulmonary edema. Cardiol J 2013; 20: 337-44.

11. Gajic O, Rana R, Winters JL, Yilmaz M, Mendez JL, Rickman OB, et al. Transfusion-related acute lung injury in the critically ill: prospective nested case-control study. Am J Respir Crit Care Med 2007; 176: 886-91.

12. Looney MR, Gropper MA, Matthay MA. Transfusion-related acute lung injury: a review. Chest 2004; 126: 249-58.

13. Vlaar AP, Binnekade JM, Prins D, van Stein D, Hofstra JJ, Schultz MJ, et al. Risk factors and outcome of transfusion-related acute lung injury in the critically ill: a nested case-control study. Crit Care Med 2010; 38: 771-8.

14. Sachs UJ. Recent insights into the mechanism of transfusion-related acute lung injury. Curr Opin Hematol 2011; 18: 436-42.

15. Menis M, Anderson SA, Forshee RA, McKean S, Johnson C, Warnock R, et al. Transfusion-related acute lung injury and potential risk factors among the inpatient US elderly as recorded in Medicare claims data, during 2007 through 2011. Transfusion 2014; 54: 2182-93.

16. Rizk A, Gorson KC, Kenney L, Weinstein R. Transfusion-related acute lung injury after the infusion of IVIG. Transfusion 2001; 41: 264-8.

17. Ganguly S, Carrum G, Nizzi F, Heslop HE, Popat U. Transfusion-related acute lung injury (TRALI) following allogeneic stem cell transplant for acute myeloid leukemia. Am J Hematol 2004; 75: 48-51.

18. Toy P, Gajic O, Bacchetti P, Looney MR, Gropper MA, Hubmayr R, et al. Transfusion-related acute lung injury: incidence and risk factors. Blood 2012; 119: 1757-67.

19. Koch CG, Li L, Sessler DI, Figueroa P, Hoeltge GA, Mihaljevic T, et al. Duration of red-cell storage and complications after cardiac surgery. N Engl J Med 2008; 358: 1229-39.

20. Silliman CC. The two-event model of transfusion-related acute lung injury. Crit Care Med 2006; 34(5 Suppl): S124-31.

21. Mangalmurti NS, Xiong Z, Hulver M, Ranganathan M, Liu XH, Oriss T, et al. Loss of red cell chemokine scavenging promotes transfusionrelated lung inflammation. Blood 2009; 113: 1158-66.

22. Curtis BR, McFarland JG. Mechanisms of transfusion-related acute lung injury (TRALI): anti-leukocyte antibodies. Crit Care Med 2006; 34(5 Suppl): S118-23.

23. Middelburg RA, van Stein D, Briët E, van der Bom JG. The role of donor antibodies in the pathogenesis of transfusion-related acute lung injury: a systematic review. Transfusion 2008; 48: 2167-76. 
24. Bux J, Sachs UJ. The pathogenesis of transfusion-related acute lung injury (TRALI). Br J Haematol 2007; 136: 788-99.

25. van Stein D, Beckers EA, Sintnicolaas K, Porcelijn L, Danovic F, Wollersheim JA, et al. Transfusion-related acute lung injury reports in the Netherlands: an observational study. Transfusion 2010; 50: 213-20.

26. Fadeyi EA, De Los Angeles Muniz M, Wayne AS, Klein HG, Leitman SF, Stroncek DF. The transfusion of neutrophil-specific antibodies causes leukopenia and a broad spectrum of pulmonary reactions. Transfusion 2007; 47: 545-50.

27. Gajic O, Gropper MA, Hubmayr RD. Pulmonary edema after transfusion: how to differentiate transfusion-associated circulatory overload from transfusion-related acute lung injury. Crit Care Med 2006; 34(5 Suppl): S109-13.

28. Jeddi R, Mansouri R, Kacem K, Gouider E, Abid HB, Belhadjali Z, et al. Transfusion-related acute lung injury (TRALI) during remission induction course of acute myeloid leukemia: a possible role for all-transretinoic-acid (ATRA)? Pathol Biol (Paris) 2009; 57: 500-2.

29. Goldberg AD, Kor DJ. State of the art management of transfusion-related acute lung injury (TRALI). Curr Pharm Des 2012; 18: $3273-84$.

30. Worsley MH, Sinclair CJ, Campanella C, Kilpatrick DC, Yap PL. Non-cardiogenic pulmonary oedema after transfusion with granulocyte antibody containing blood: treatment with extracorporeal membrane oxygenation. Br J Anaesth 1991; 67: 116-9.

31. Moore SB. Transfusion-related acute lung injury (TRALI): clinical presentation, treatment, and prognosis. Crit Care Med 2006; 34(5 Suppl): S114-7.

32. Kleinman S, Caulfield T, Chan P, Davenport R, McFarland J, McPhedran S, et al. Toward an understanding of transfusion-related acute lung injury: statement of a consensus panel. Transfusion 2004; 44: 1774-89.

33. Chapman CE, Stainsby D, Jones H, Love E, Massey E, Win N, et al. Ten years of hemovigilance reports of transfusion-related acute lung injury in the United Kingdom and the impact of preferential use of male donor plasma. Transfusion 2009; 49: 440-52.

34. Ozier Y, Muller JY, Mertes PM, Renaudier P, Aguilon P, Canivet N, et al. Transfusion-related acute lung injury: reports to the French Hemovigilance Network 2007 through 2008. Transfusion 2011; 51: 2102-10.

35. Su L, Kamel H. How do we investigate and manage donors associated with a suspected case of transfusion-related acute lung injury. Transfusion 2007; 47: 1118-24.

36. Eder AF, Herron R, Strupp A, Dy B, Notari EP, Chambers LA, et al. Transfusion-related acute lung injury surveillance (2003-2005) and the potential impact of the selective use of plasma from male donors in the American Red Cross. Transfusion 2007; 47: 599-607.

37. Carrick DM, Norris PJ, Endres RO, Pandey S, Kleinman SH, Wright D, et al. Establishing assay cutoffs for HLA antibody screening of apheresis donors. Transfusion 2011; 51: 2092-101.

38. Triulzi DJ, Kleinman S, Kakaiya RM, Busch MP, Norris PJ, Steele WR, et al. The effect of previous pregnancy and transfusion on HLA alloimmunization in blood donors: implications for a transfusion-related acute lung injury risk reduction strategy. Transfusion 2009; 49: 1825-35.

39. Tuinman PR, Vlaar AP, Cornet AD, Hofstra JJ, Levi M, Meijers JC, et al. Blood transfusion during cardiac surgery is associated with inflammation and coagulation in the lung: a case control study. Crit Care 2011; 15: R59.

40. Clifford L, Jia Q, Subramanian A, Yadav H, Wilson GA, Murphy SP, et al. Characterizing the epidemiology of postoperative transfusionrelated acute lung injury. Anesthesiology 2015; 122: 12-20.

41. Carson JL, Sieber F, Cook DR, Hoover DR, Noveck H, Chaitman BR, et al. Liberal versus restrictive blood transfusion strategy: 3-year survival and cause of death results from the FOCUS randomised controlled trial. Lancet 2014 [Epub ahead of print]

42. Eder AF, Herron RM Jr, Strupp A, Dy B, White J, Notari EP, et al. Effective reduction of transfusion-related acute lung injury risk with malepredominant plasma strategy in the American Red Cross (2006-2008). Transfusion 2010; 50: 1732-42. 